\begin{tabular}{l|l} 
REVISTA & $\begin{array}{l}\text { Revista Educación } \\
\text { ISSN: } 0379-7082 \\
\text { ISSN: 2215-2644 } \\
\text { revedu@gmail.com } \\
\text { Universidad de Costa Rica } \\
\text { Costa Rica }\end{array}$
\end{tabular}

\title{
Las variables como elemento sustancial en el método científico
}

\author{
Arroyo Valenciano, Juan Antonio \\ Las variables como elemento sustancial en el método científico \\ Revista Educación, vol. 46, núm. 1, 2022 \\ Universidad de Costa Rica, Costa Rica \\ Disponible en: https://www.redalyc.org/articulo.oa?id=44068165026 \\ DOI: https://doi.org/10.15517/revedu.v46i1.45609
}

\section{(c) $\odot \Theta \Theta$}

Esta obra está bajo una Licencia Creative Commons Atribución-NoComercial-SinDerivar 3.0 Internacional. 


\title{
Las variables como elemento sustancial en el método científico
}

\author{
Variables as a Substantial Element of the Scientific Method
}

Juan Antonio Arroyo Valenciano

Universidad de Costa Rica, Costa Rica

juan.arroyo@ucr.ac.cr

DOI: https://doi.org/10.15517/revedu.v46i1.45609 Redalyc: https://www.redalyc.org/articulo.oa? $\mathrm{id}=44068165026$

(iD) https://orcid.org/0000-0002-3092-3872

Recepción: 03 Marzo 2021

Aprobación: 16 Junio 2021

\section{ReSUMEN:}

La comunidad científica coincide en que tanto el problema como la hipótesis no solo constituyen dos fases relevantes del método científico, sino que, en su esencia misma, comparten un elemento común y sustancial: las variables. Por tanto, el éxito y la precisión que requiere una investigación, para obtener conocimiento válido y conclusiones confiables en el campo de la educación, dependerá de la rigurosidad con que cada variable sea definida. Con el fin de identificar los aportes que las fuentes documentales establecen para que tal rigurosidad se cumpla, se desarrolló una investigación teórico documental, que definió como unidades de estudio a las principales obras sobre introducción y metodología de la investigación, en general, y, en específico, para el campo de la educación, que fueron publicadas en el período comprendido entre el año 2000 y 2020. La investigación determinó que las unidades de estudio reconocen la relevancia de las variables en la estructura, tanto del problema como de la hipótesis. La presentación que hacen del tema de las variables resulta ser referencial, supeditada a destacar su importancia. En cuanto a su definición, enfatizan en su enunciado: constitutivo y operativo, sin trascender a plantear los aspectos, normas o considerandos que debe seguir la persona investigadora en educación para concretar tales definiciones. Ante dichos resultados, este ensayo tiene por objetivo proponer un modelo de estructura conceptual tridimensional, que faculte a la persona investigadora en delimitar el significado constitutivo, operativo

Palabras ClaVe: Investigación educativa, Variables, Operacionalización de variables, Método científico.

\section{ABSTRACT:}

The scientific community acknowledges that both the problem and hypothesis not only comprise two relevant phases of the scientific method, but also share a common, more substantial element that conforms its very essence - variables. The success and precision required in order to obtain valid knowledge and reliable conclusions in the field of education depends on the rigor used to define such variables. A document-based theoretical study of sources in the field of education adhering to this research methodology and published between 2000 and 2020 was conducted in order to determine if the conditions of the scientific method were met. This study acknowledge that such variables are an intrinsic part of the structure of both the problem and hypothesis. Variables serve as references and are an essential part of the operative statement without contemplating norms or considerations that the researchers must adhere to when establishing such definitions. This study, therefore, presents a threedimensional conceptual model, that empowers researchers to delimit essential operative and instrumental meanings of variables as a substantial element of the scientific method.

KeYworDs: Educational Research, Variables, Operational Variables, Scientific Method.

\section{INTRODUCCIÓN}

Aun cuando existen diversas tendencias con respecto al concepto de ciencia y a las fases del método científico como proceso sistemático, mediante el cual se puede obtener el conocimiento, existe coincidencia entre la comunidad científica que el planteamiento del problema y la formulación de las hipótesis son dos fases clave de este proceso.

Con respecto al planteamiento del problema, los aportes de Kerlinger (2000) señalan que este constituye el punto de partida de toda investigación científica. Su formulación implica una delimitación clara de lo que se va a investigar y en su enunciado se prevé la expresión de una relación entre variables. Para Ary et al. (2010) 
el problema indaga la relación existente entre dos o más variables. Baena (2017) considera al problema como una proposición que expresa con claridad las variables de la investigación científica.

En relación con la formulación de la hipótesis, Arellano (1990) enuncia que esta predice la correlación entre variables y es, en esencia, la aplicación de una teoría particular a las variables de un estudio concreto. Para Ávila (2006) representa un proyecto de respuesta al problema a partir de una presunción sobre la relación entre variables. Ary et al. (2010) consideran que la hipótesis "debe señalar la relación que se espera entre las variables" (p. 86). Guadarrama (2012) establece que la hipótesis es una respuesta al problema, que surge de la predicción generada de la relación dada entre variables. García (2016) la delimita como una conjetura, que se expresa como un enunciado afirmativo y que enlaza al menos dos variables. Finalmente, para Monroy y Nava (2018) las hipótesis “identifican o explicitan las variables objeto del estudio" (p.85).

Los señalamientos planteados fortalecen la noción de que problema e hipótesis no solo constituyen dos fases relevantes del método científico, sino que comparten, en su esencia misma, un elemento común: las variables. En este orden de ideas, cabe destacar que Zorrilla y Torres (1992) esbozan que "la investigación científica gira alrededor de las variables, debido a que la finalidad del trabajo científico es descubrir la existencia de ellas y su magnitud, así como probar las relaciones que las unen entre sí” (p.62). Pérez (2007) llega a concebirlas como "la unidad fundamental del método de la investigación científica" (p.176). Mientras que Espinoza (2018) las destaca como el concepto de vital importancia para la investigación, Pérez et al. (2020) abogan que, en la investigación, el problema debe reducirse a su mínima expresión: las variables, para luego integrarlas y relacionarlas unas con otras en la hipótesis.

Por lo expuesto, es posible señalar que el éxito y la precisión que requiere una investigación, para obtener conocimiento válido y conclusiones confiables sobre un determinado objeto de estudio, dependen de la rigurosidad con que cada una de las variables presentes, tanto en el problema como en la hipótesis de investigación, sean definidas para concretar su identificación, observación y análisis.

Con el fin de determinar los aportes que las fuentes documentales establecen para que tal rigurosidad se cumpla, se procedió desarrollar una investigación teórico documental de las principales obras publicadas sobre introducción y metodología de la investigación durante el período comprendido entre el año 2000 y 2020.

Para establecer un marco de comparación se usó el método descriptivo, a partir del cual se establecieron tres aspectos relevantes para desarrollar el análisis documental de cada una de las obras, a saber: el primero, determinar si la obra reconocía de forma explícita o implícita la relevancia de las variables y su relación intrínseca con el problema y la hipótesis de investigación; el segundo, identificar el tratamiento dado al desarrollo del tema de las variables; finalmente, el tercero, establecer la forma o las dimensiones señaladas para su definición.

La indagación consideró como sus unidades de estudio las obras de investigación propuestas por Kerlinger (2000), Barrantes (2002), McMillan y Schumacher (2005), Salinas y Cárdenas (2008), Gómez (2009), Bisquerra (2009), Ary et al. (2010), Hernández et al. (2010), Pérez et al. (2012), Ackerman y Com (2013), Cruz et al. (2014), García (2016), Baena (2017), Monroy y Nava (2018) y Pérez et al. (2020).

$\mathrm{El}$ análisis teórico documental desarrollado permitió, según cada uno de los aspectos relevantes y rectores del análisis, establecer los siguientes resultados:

- De forma explícita o implícita, las obras de investigación analizadas reconocen la relevancia de las variables, tanto en la estructura del problema como de la hipótesis, con lo que se confirma su relación intrínseca. Se identifica una forma de consenso en cuanto a que sin su definición no se podría originar la investigación científica.

- El desarrollo dado al tema de las variables se caracteriza por ser simple, referencial y superficial. Se identifica que, por lo general, queda supeditado a destacar la importancia de definirlas, clasificarlas y determinar su tipología. Todas las unidades de estudio hacen alusión a las variables independientes y dependientes. Por su parte, las unidades de estudio de Kerlinger (2000), Ary et al. (2010) y García (2016) 
agregan las variables discretas y continuas. Mientras que Salinas y Cárdenas (2008), Bisquerra (2009), Pérez et al. (2012), García (2016), Monroy y Nava (2018) proponen otras variables, a saber: perturbadoras, intervinientes, ajenas, extrañas, ambientales y personales. También, algunas unidades de estudio hacen referencia a variables continuas y discontinuas, dicotómicas y categóricas, activas y de atributo; entre ellas las de McMillan y Schumacher (2005), Ackerman y Com (2013), Cruz et al. (2014).

- Las obras son coincidentes en destacar que las variables deben ser definidas de forma teórica, constitutiva o conceptual, esto mediante elaboraciones teóricas relacionadas con su estructura y funcionamiento, que permitan describirlas en su esencia a partir del uso de conceptos. Son también concurrentes en plantear que deben ser definidas de forma operativa u operacional, llevarlas de la teoría al dato mediante la especificación de los procedimientos, operaciones y actividades que la harán observable para poder medirla y clasificarla. Solo una unidad de estudio, la de Barrantes (2002), alude que las variables deben ser definidas de forma instrumental, lo cual asocia a definir y elaborar los instrumentos, a través de los cuales se recolectará la evidencia que hará observable a la variable. No obstante, ninguna unidad de estudio llega a un planteamiento sobre los procedimientos, normas o considerandos que deben orientar y asesorar el trabajo de la persona investigadora, para poder definirlas desde estas tres dimensiones.

Los resultados del análisis teórico documental coinciden con lo planteado por Piñero y Perozo (2020) cuando indican que en la literatura científica las variables son representadas como conceptos, los cuales, por su contenido superficial, no permiten distinguir sus elementos, de manera que limitan las opciones para establecer los procedimientos requeridos para su definición.

Por lo expuesto, el presente ensayo tiene como objetivo proponer un modelo de estructura conceptual tridimensional que faculte a la persona investigadora en educación a delimitar el significado constitutivo, operativo e instrumental de las variables como elemento sustancial en el método científico.

Para ello, se organizan sus planteamientos conforme a los siguientes apartados: Noción de Variable, en el que se realiza el estudio del concepto, el cual permite concluir con un planteamiento puntual de su significado; Modelo de Estructura Conceptual Tridimensional de la Variable, en el cual se establecen y definen las dimensiones que debe alcanzar una variable para ser investigada con mayor exactitud y precisión, para continuar con el apartado Discusión y finalizar con el de Conclusiones.

Finalmente, es importante señalar que en este ensayo se hace uso de la palabra cosa, la cual se delimita como el ente abstracto o concreto que origina el asunto o cuestión que motiva la acción de investigar. Cosa como tal puede asumir la identidad de persona, constructo o de un fenómeno natural o social.

\section{Noción de VARIABLE}

La palabra variable proviene del latín y está constituida por dos componentes léxicos: variare que significa cambiar de aspecto y able que establece la posibilidad de. Por consiguiente, en sentido exacto conforme a su etimología, variable se concibe como la posibilidad de cambiar de aspecto. Lo expuesto conduce al planteamiento de una primera interrogante por resolver, para precisar el significado del concepto variable: ¿Qué significa cambiar de aspecto? Si cambiar significa modificar o reemplazar una cosa por otra y aspecto significa la visión con que se observa o contempla una cosa, entonces, una cosa es variable cuando tiene la posibilidad de experimentar modificaciones o sustituciones por medio de las cuales su identidad se percibe u observa de forma diferente.

A partir de lo señalado surge la búsqueda de precisar el significado del concepto variable desde una segunda interrogante: ¿Qué es la identidad de la cosa y qué modificaciones o sustituciones puede experimentar? La identidad de la cosa está constituida por todos aquellos rasgos propios que posee. La identidad tiene la doble función de caracterizar a la cosa como única en su singularidad y a la vez común ante las demás de su género. Con respecto a las modificaciones o sustituciones que la identidad de la cosa puede experimentar, están las modificaciones perceptibles o tangibles que actúan sobre los rasgos visibles de la cosa, es decir, 
sus características, cuya detección se realiza con facilidad a nivel de experiencia sensorial. Las sustituciones pueden ser imperceptibles o intangibles y actuar sobre rasgos invisibles a nivel de experiencia sensorial, es decir, sobre las cualidades, por lo que se requiere de modelos e instrumentos diseñados para hacerlos perceptibles.

Lo analizado permite precisar el significado del concepto variable como aquella modificación en una característica o cualidad, perceptible o imperceptible que muda la identidad de la cosa, origen del asunto o cuestión que motiva la acción de investigar.

\section{MODELO DE ESTRUCTURA CONCEPTUAL TRIDIMENSIONAL DE LA VARIABLE}

El modelo de estructura conceptual tridimensional de la variable fundamenta su relevancia en los siguientes tres planteamientos: el primero establece que las variables son elemento esencial de dos de las fases del método científico, como lo son el planteamiento del problema y la construcción de hipótesis. Sobre ellas gravitan las demás fases, a saber: deducción de consecuencias, prueba de hipótesis e introducción de conclusiones en la teoría; conforme a las grandes líneas que Bunge (2013) estima, sigue el método científico, como guía para evitar que la investigación se pierda en el caos de los fenómenos y la persona investigadora perezca por el sortilegio de sus prejuicios.

El segundo determina, según Baena (2017), que "las variables son instrumentos de análisis que conforman las categorías a un nivel manifiesto de la realidad" (p. 93), con ello se logra constituir un puente entre esta y la teoría.

El tercero, que la investigación teórico documental realizada de las principales obras publicadas sobre introducción y metodología de la investigación, no consideran en sus planteamientos los aspectos, normas o considerandos que requiere la persona investigadora en educación para desarrollar una definición de variables, que, según los referentes empíricos, alcance mayor exactitud y precisión (Pérez et al., 2020).

Ante tales planteamientos, con el fin de atender tal requerimiento, se propone el modelo de estructura conceptual tridimensional de la variable, el cual facilitará a la persona investigadora en el campo de la educación a fijar y explicar la característica o cualidad, tangible o intangible, que muda la identidad de la cosa desde la dimensión constitutiva, operativa e instrumental, las cuales se particularizan a continuación .

\section{Dimensión constitutiva}

La dimensión constitutiva tiene por finalidad definir la variable desde la relación definiendum-definiens, es decir, desde la correspondencia entre lo que se define y el conjunto de términos a los que recurre la persona investigadora que son necesarios para describir semánticamente a la variable en su esencia. Una interpretación de los aportes señalados por Copi y Cohen (2013) y Gianella (2010) permite establecer que para la definición de una variable, desde la dimensión constitutiva deben cumplirse tres condiciones básicas:

- Exponer el significado de la variable sin usarla como término de su propia definición.

- Enunciar el significado con base en las características y cualidades esenciales que mejor representan la mutación que experimenta la identidad de la cosa.

- Determinar que el significado dado a la variable sea preciso, esto es, que no exceda o carezca en su enunciado de las características o cualidades esenciales de esta.

Las condiciones básicas enunciadas determinan que la definición constitutiva expresa las características y cualidades esenciales que mutan en la identidad de la cosa. Sin embargo, existen mutaciones de identidad cuyas características o cualidades son difíciles de identificar o representar, por lo que se debe recurrir a enunciados que revelan la forma en que estas se manifiestan. La facilidad o dificultad con que se puedan 
expresar las características o cualidades que mutan en la cosa, origina dos categorías de definición constitutiva de una variable, a saber:

- Designativas: definen la variable haciendo uso de enunciados que expresan sus características o cualidades.

- Denotativas: definen la variable por medio de enunciados a través de los cuales se revela o manifiesta.

A la vez, cada categoría suscita, según corresponda, subcategorías de definición constitutiva de una variable; así, dentro de las Designativas están las siguientes:

- Lexicográficas: utilizan un lenguaje natural para enunciar el significado de la variable, lo cual puede dar margen a la vaguedad y a la ambigüedad.

- Estipulativas: manifiestan el significado de la variable en forma convencional a un término nuevo o al que ya poseía otro significado.

- Aclaratorias: expresan el significado de la variable a partir del significado lexicográfico con una mayor precisión de términos para eliminar la ambigüedad y vaguedad.

- Teóricas: exponen el significado de la variable mediante el uso del vocabulario propio de una determinada teoría.

- Funcionales: explican el significado de la variable en términos de su función y no por la forma.

Por otra parte, dentro de la Denotativas, aparecen:

- Ostensivas: definen la variable a partir de casos particulares a los que corresponde esta, para lo cual se apoya en elementos del entorno y en relaciones de semejanza mediante el uso de analogías.

- Enumerativas: definen la variable a partir de la inclusión, cita o enlistado de los términos o elementos que forman parte de la variable.

La Tabla 1 presenta un sumario de las categorías y subcategorías descritas; con énfasis en sus características plantea un ejemplo relacionado con la definición constitutiva de la variable estudiante.

TABLA 1

Categorías y subcategorías de la definición constitutiva

\begin{tabular}{l|l|l|l}
\hline Categoria & Subcategoría & Característica & Variable: Estudiante \\
\hline & $\begin{array}{l}\text { Lexicográficas } \\
\text { Estipulativas } \\
\text { Aclaratorias }\end{array}$ & $\begin{array}{l}\text { Usar un lenguaje natural. } \\
\text { Dar significado convencional. } \\
\text { Restringir la vaguedad y ambigüedad. }\end{array}$ & $\begin{array}{l}\text { Persona que asiste a un centro educativo para aprender. } \\
\text { Persona que aprende. Escolar } \\
\text { Persona dedicada a la aprehensión de los conocimientos afines a un } \\
\text { determinado arte o disciplina científica. } \\
\text { Persona que mediante un proceso planificado de enseñanza y } \\
\text { aprendizaje adquiere y comprende los conocimientos definidos en } \\
\text { un plan de estudios para alcanzar el desarrollo de sus capacidades, } \\
\text { destrezas y facultades intelectuales, morales y de comportamiento } \\
\text { humano. } \\
\text { Persona que estudia. }\end{array}$ \\
\hline Deóricas & Euncionar un lenguaje asociado a una teories & Proporcionar el significado por función. & $\begin{array}{l}\text { La persona que, como Antonio, recibe lecciones en una escuela. } \\
\text { Persona que recibe lecciones, lee, escribe, prepara tareas y desarrolla } \\
\text { pruebas. }\end{array}$ \\
\hline
\end{tabular}

Fuente: elaboración propia.

\section{Dimensión operativa}

Concretada la definición constitutiva de la variable según corresponda a una categoría y subcategoría, la persona investigadora debe proceder a definirla desde la dimensión operativa. Es decir, desde el conjunto de 
operaciones empíricas, indicadores o situaciones mediante las cuales se podrá observar, identificar, medir y valorar la mutación que experimenta la característica o cualidad de la cosa. Al respecto Arias-Gómez et al. (2016) señalan que la definición operativa establece los hechos observables y objetivos, claros y explícitos que evitan la ambigüedad y aseguran su significado unívoco.

A partir de las ideas expuestas por Brenes (2003) y Espinoza (2018), la definición operacional o proceso de transformación de la variable debe tener términos concretos, observables y medibles, y considerar las siguientes cinco condiciones:

- Delimitar las normas cualitativas y cuantitativas a través de las cuales se identificará la presencia y se establecerá la magnitud del cambio o modificación experimentada por los rasgos de la cosa.

- Definir los datos que se requieren para constatar con exactitud las modificaciones o cambios dados en los rasgos de la cosa.

- Delimitar el conjunto de cosas que serán observadas para identificar, medir y valorar las modificaciones o cambios, así como el espacio donde se ubican para realizar la toma de datos.

- Diseñar los patrones o categorías de análisis para la clasificación de la cosa según la mutación experimentada en una de sus características o cualidades. Si se está ante un rasgo cualitativo, a partir de su presencia o ausencia, se podrán establecer dos categorías de clasificación: dicotómica o politómica. Si el rasgo es cuantitativo, a partir de su magnitud podría ser clasificado como continuo, si puede asumir cualquier valor dentro de una determinada escala infinita. $O$ discontinuo porque solo puede tomar un valor fijo en la escala finita utilizada para su medición.

- Elaborar los modelos de análisis mediante los cuales se estudia los cambios experimentados en los rasgos de la cosa, para establecer así proposiciones o afirmaciones finales sobre tales mutaciones con apoyo de la teoría.

Las condiciones expuestas extractan que la definición operativa de una variable debe considerar en su estructura al tipo de rasgo a estudiar, y a partir de este establecer las normas de medición según corresponda a modo o magnitud, lo que llevará a identificar con mayor claridad la clase de datos que se requieren y, por ende, identificar el conjunto de cosas según su naturaleza y de donde se deben obtener, para continuar con el establecimiento de los patrones y el tipo de análisis que se desarrollará. La Tabla 2 permite precisar tal estructura y sus elementos.

TABLA 2

Elementos de la estructura de la definición operativa

\begin{tabular}{|c|c|c|c|c|c|}
\hline Rasgo & \begin{tabular}{|l} 
Norma de \\
medición
\end{tabular} & Datos & $\cos a$ & Patrón & Análisis \\
\hline \multirow{2}{*}{ Cualitativo } & \multirow{2}{*}{ Modo o aspecto } & \multirow{2}{*}{$\begin{array}{l}\text { Para } \\
\text { identificar y } \\
\text { ordenar } \\
\end{array}$} & \multirow{4}{*}{$\begin{array}{l}\text { Abstracta } \\
\text { Concreta }\end{array}$} & Dicotómica & Nominal \\
\hline & & & & Politómica & Ordinal \\
\hline \multirow{2}{*}{ Cuantitativo } & \multirow{2}{*}{$\begin{array}{l}\text { Magnitud o } \\
\text { tamaño }\end{array}$} & \multirow{2}{*}{$\begin{array}{l}\text { Para establecer } \\
\text { diferencias }\end{array}$} & & Continua & Intervalo \\
\hline & & & & Discontinua & Razón \\
\hline
\end{tabular}

Fuente: elaboración propia.

En resumen, la dimensión operativa representa la expresión numérica de la variable, a través de la cual se busca darle un significado a esa medición, ya sea por su presencia en modo o magnitud. Esta asignación numérica permite, por tanto: determinar entre en el conjunto de cosas condiciones de igualdad y de diferenciación, con respecto al rasgo que muta, para poder establecer su orden y clasificación. 


\section{Dimensión instrumental}

Las definiciones dadas a la variable en la dimensión constitutiva y operativa constituyen la base para proceder con la definición instrumental, al establecer la relación entre estas y la elaboración de los instrumentos requeridos para realizar la recolección de los datos que, una vez analizados, fundamentarán si el conjunto de cosas en estudio experimentó una modificación o cambio en uno de sus rasgos.

La definición instrumental tiene por función facilitar el registro completo y detallado de la mutación que experimenta la entidad de la cosa, en ocasiones no sensible a ser captada de forma sensorial; esto facilita su cuantificación o cualificación.

Para lograr tal cuantificación o cualificación del rasgo que muda, la definición instrumental elabora un conjunto de enunciados o interrogaciones, que integrados y relacionados de forma lógica, conforman un instrumento, mediante el cual la persona investigadora procederá a la recolección de los datos, para determinar la presencia, el modo y la magnitud de la variable. Por tanto, cada enunciado o interrogación, en lo individual y, por ende, en el instrumento como un todo, debe cumplir con dos requisitos fundamentales, a saber:

- Garantizar que al medir la misma variable más de una vez los datos obtenidos permitirán obtener resultados similares.

- Medir el rasgo que debe medir con exactitud y veracidad, esto afín al rasgo en estudio y congruente con la definición constitutiva de la variable.

La Tabla 3 presenta una síntesis de los instrumentos básicos que la persona investigadora puede diseñar o utilizar para desarrollar la definición instrumental de la variable.

TABLA 3

Instrumentos para desarrollar la definición instrumental

\begin{tabular}{l|l}
\hline Instrumento & Énfasis \\
\hline Encuesta & $\begin{array}{l}\text { Determinar aspectos como creencias, hábitos, intenciones y } \\
\text { opiniones. }\end{array}$ \\
\hline Escala & $\begin{array}{l}\text { Medir la predisposición (actitud) ante algo. Determinar la } \\
\text { dirección (positiva o negativa) y la intensidad (alta y baja). }\end{array}$ \\
\hline Hoja de cotejo & $\begin{array}{l}\text { Enlistar los aspectos a observar en el conjunto de cosas } \\
\text { relacionadas con el rasgo que muta. }\end{array}$ \\
\hline
\end{tabular}

Fuente: elaboración propia.

Para finalizar, la Tabla 4, denominada Matriz del modelo de estructura conceptual tridimensional de la variable, extracta los elementos que integran a cada una de las dimensiones del modelo de estructura tridimensional propuesto para la definición de la variable. 
TABLA 4

Matriz del modelo de estructura conceptual tridimensional de la variable

\begin{tabular}{|c|c|c|c|c|}
\hline Dimensiones de la definici & & & & \\
\hline Constitutiva & & Operativa & & Instrumental \\
\hline $\begin{array}{l}\text { Categoría: } \\
\text { Denotativa }\end{array}$ & Designativa & $\begin{array}{l}\text { Rasgo: } \\
\text { Cuantitativo }\end{array}$ & Cualitativo & $\begin{array}{l}\text { Diseño: } \\
\text { Enunciados }\end{array}$ \\
\hline $\begin{array}{l}\text { Subcategorías: } \\
\text { Ostensivas Enumerativas }\end{array}$ & Lexicográficas Estipulativas Aclaratorias Teóricas Funcionales & $\begin{array}{l}\text { Norma: } \\
\text { Magnitud }\end{array}$ & Modo & $\begin{array}{l}\text { Requerimiento: } \\
\text { Confiabilidad }\end{array}$ \\
\hline & & $\begin{array}{l}\text { Datos: } \\
\text { Diferenciar }\end{array}$ & Ordenar & $\begin{array}{l}\text { Instrumentos a desarrollar: } \\
\text { Encuestas Escalas Hojas de cotejo }\end{array}$ \\
\hline & & $\begin{array}{l}\text { Cosa: } \\
\text { Abstracta o concreta }\end{array}$ & & \\
\hline & & $\begin{array}{l}\text { Patrón: } \\
\text { Continua Discontinua } \\
\end{array}$ & Dicotómica Politómica & \\
\hline & & $\begin{array}{l}\text { Análisis: } \\
\text { Intervalo Razón }\end{array}$ & Nominal Ordinal & \\
\hline
\end{tabular}

Fuente: elaboración propia.

\section{Discusión}

La investigación teórico documental de las principales obras editadas sobre introducción y metodología de la investigación, durante el período comprendido entre el año 2000 y 2020, evidencia un enfoque parcial y limitado con respecto a la definición de las variables como elemento sustancial en el método científico.

Lo identificado obliga a la reflexión y a un replanteamiento de tal enfoque, con la finalidad de proponer un modelo de estructura conceptual tridimensional de la variable, que, integrando las dimensiones constitutiva, operativa e instrumental, oriente a la persona investigadora en la construcción y desarrollo de una definición apropiada, congruente y precisa de las variables que se propone investigar.

Es necesario comprender que la precisión y rigurosidad con que sean definidas las variables, conforme al modelo de estructura conceptual tridimensional propuesto, permitirá a la persona investigadora en el campo de la educación una aproximación concreta y coherente de la realidad del fenómeno educativo en estudio. Además, le asegura un mayor grado de comprensión, tanto de su naturaleza y estructura, como de su comportamiento o funcionamiento. A partir de tal comprensión, se pretende validar o actualizar el conocimiento vigente y fundamentar con este los procesos de toma de decisiones para la atención y solución de los problemas que se presentan en el campo de la educación.

El modelo de estructura conceptual tridimensional propuesto no debe ser considerado como definitivo o acabado, todo lo contrario, se presenta como marco de referencia para que la persona investigadora, logre mayor objetividad, precisión y rigor científico en la definición de las variables. Ante todo, pretende exaltar el interés por el estudio y el desarrollo de la investigación en un área del conocimiento reconocida como relevante, pero apenas explorada: las variables como elemento sustancial en el método científico.

\section{Conclusiones}

De acuerdo con el análisis documental de las obras de introducción y metodología de la investigación, la interpretación de los conceptos, y el modelo de estructura conceptual tridimensional propuesto para la definición de variables, se concluye que:

- Las variables constituyen el elemento esencial y común de dos fases del método científico: planteamiento del problema y la formulación de las hipótesis.

- Las obras de introducción y metodología de la Investigación, reconocen la relevancia que tienen las variables como elemento sustancial al método científico. No obstante, aun cuando aluden a la importancia de definirlas, lo expresan en función de su dimensión constitutiva y operativa, pero de una forma superficial, sin establecer procedimientos concretos para tal fin y omite la dimensión instrumental. 
- El modelo de estructura conceptual tridimensional planteado pretende dar un aporte a la investigación científica en el campo de la educación que faculte a la persona investigadora a diseñar la descripción semántica de la variable en su esencia, determinar su significado cuantitativo o cualitativo a partir de su apariencia en modo o magnitud, y, finalmente, ajustar o elaborar los instrumentos para acceder al registro de su comportamiento o funcionamiento.

En coda, si la investigación científica en educación, a través de su método, promueve de forma ordenada y sistemática la indagación, estudio y análisis riguroso de una determinada situación fáctica relacionada con su objeto de estudio, con el explícito fin de acreditarlo o no, la definición precisa de las variables es, por tanto, esencial para tal acreditación, que subsecuentemente conducirá a ampliar y desarrollar tanto la teoría como el conocimiento científico en el campo de la educación.

\section{Referencias Bibliográficas}

Ackerman, S. y Com, S. (2013). Metodologia de la Investigación. Aula Taller.

Arellano, J. (1990). Elementos de Investigación. EUNED.

Arias-Gómez, J., Villasís-Keever M. y Miranda-Novales, M. (2016). El protocolo de investigación IV: las variables de estudio. Alergia México, 63(3), 303-310. https://www.redalyc.org/articulo.oa?id=486755025003

Ary, D., Jacobs, L. y Razavieh, A. (2010). Introduction to Research in Education. [Introducción a la Investigación Pedagógica]. Cengage Learning.

Ávila, H. (2006). Introducción a la metodología de la investigación. Cuauhtémoc. http://www.eumed.net/libros/200 $6 c / 203 /$

Baena, G. (2017). Metodología de la investigación. Patria.

Barrantes, R. (2002). Investigación camino al conocimiento. EUNED.

Bisquerra, R. (2009). Metodología de la Investigación Educativa. La Muralla.

Brenes, A. (2003). Los trabajos finales de graduación: su elaboración y presentación en las Ciencias Sociales. EUNED.

Bunge, M. (2013). La ciencia, su método y su filosofia. Laetoli.

Copi, I. y Cohen, C. (2013). Introducción a la Lógica. Limusa.

Cruz, C., Olivares, S y García, M. (2014). Metodología de la Investigación. Patria.

Espinoza, E. (2018). Las variables y su operacionalización en la investigación educativa. Parte I. Revista Conrado, 14(65), 39-49. https://conrado.ucf.edu.cu/index.php/conrado/article/view/814/845

García, J. (2016). Metodología de la Investigación para Administradores. Ediciones de la U.

Gianella, A. (2010). Introducción a La Epistemología y a la Metodología de la Ciencia. De la Campana.

Gómez, M. (2009). Introducción a la Metodología de la Investigación Cientifica. Brujas.

Guadarrama, P. (2012) Dirección y asesoría de la investigación cientifica. Editorial Ciencias Sociales.

Hernández, R., Fernández, C. y Baptista, M. (2010). Metodología de la Investigación. Mc. Graw Hill.

Kerlinger, F. (2000). Investigación del comportamiento técnicas y metodología. McGraw Hill Interamericana.

McMillan, J. y Schumacher, S. (2005). Investigación Educativa. Pearson Educación.

Monroy, M. y Nava, N. (2018). Metodología de la Investigación. Lapislázuli.

Pérez, J. (2007). Las variables en el método científico. Revista de la Sociedad Quimica del Perú, 73(3), 171-177.

Pérez, R., Galán, A. y Quintanal, J. (2012). Métodos y Diseños de Investigación en Educación. UNED.

Pérez, R., Pérez, L. y Seca, M. (2020). Metodología de la Investigación Cientifica. Maipue.

Piñero, L. y Perozo, L. (2020). Construcción teórica: sinónimo, definición operacional y sistematización de variables educativas. Revista Cientifica Electrónica de Ciencias Humanas, 16(47), 16-30. http://www.revistaorbis.org/pd $\mathrm{f} / 51 / \mathrm{art} 2 . \mathrm{pdf}$

Salinas, P. y Cárdenas, M. (2008). Métodos de Investigación Social. Universidad Católica. 
Zorrilla, A. y Torres, M. (1992). Guia para Elaborar la Tesis. McGraw Hill.

\section{INFORMACIÓN ADICIONAL}

Cómo citar: Arroyo-Valenciano, J. A. (2022). Las variables como elemento sustancial en el método científico. Revista Educación, 46(1). http://doi.org/10.15517/revedu.v46i1.45609 